\title{
Estrategias colaborativas de aprendizajes de estudiantes durante la COVID-19. Caso: Curso de Teoría de Control
}

\section{Collaborative student learning strategies during COVID-19. Case: Control Theory Course}

\author{
Eugenio Navarro Zeballos ${ }^{1}$, Ignacio Chang ${ }^{2 *}$ \\ ${ }^{1}$ Licenciatura en Ingeniería Electromecánica, Facultad de Ingeniería Eléctrica, Universidad Tecnológica de Panamá \\ ${ }^{2}$ Facultad de Ingeniería Eléctrica, Grupo SCIII, Universidad Tecnológica de Panamá
}

\begin{abstract}
Resumen Gracias al amplio desarrollo y popularización de las redes informáticas, el conocimiento se encuentra al alcance de prácticamente de cualquier persona al instante. La educación actual continúa con métodos del siglo XIX y XX. Por esta razón, los métodos de enseñanza en la educación superior deben ser actualizados a la realidad que se vive para tratar de potenciar las capacidades del estudiante. Este documento presenta un caso de estudio donde se busca determinar cualitativamente la influencia de la utilización de métodos de aprendizaje que incentivan el trabajo colaborativo en los estudiantes. Por lo que, se le asignó a cada grupo de estudiantes la tarea de resolver un problema de aplicación sobre el curso dado, y adicional se introdujo un reto extra, en el cual se podía utilizar cualquier herramienta libre. El objetivo principal era explicar de manera didáctica cómo se resolvería el problema, con la finalidad de reforzar los conocimientos previos del curso, así como desarrollar habilidades blandas como el trabajo en equipo y la creatividad. Los resultados obtenidos sugieren que utilizar el método colaborativo estimula el interés de los estudiantes y en participar; facilita organizar su tiempo para la solución; permite coordinar con los demás miembros del grupo y ser autodidacta.
\end{abstract}

Palabras clave Ambiente de aprendizaje, aprendizaje colaborativo, estudio de casos, herramientas de aprendizaje y metodología.

\begin{abstract}
Thanks to the extensive development and popularization of computer networks, knowledge is available to virtually anyone at any time, however, today's education continues with 19th and 20th century methods. For this reason, the teaching methods in higher education must be updated to the reality that exists to try to enhance the student's abilities. This document presents a case study where it seeks to qualitatively determine the influence of the use of learning methods that encourage collaborative work in students. For this, each group of students was assigned the task of solving an application problem on the given course, and an additional challenge was introduced, in which any free tool could be used. The main objective was to explain in a didactic way how the problem would be solved, in order to reinforce the previous knowledge of the course, as well as develop soft skills such as teamwork and creativity. The results obtained suggest that using the collaborative method stimulates students' interest in participating, makes it easier to organize their time for the solution, allows them to coordinate with the other members of the group and be self-taught.
\end{abstract}

Keywords Case studies, collaborative learning, learning environment, learning tools, methodology.

* Corresponding author: ignacio.chang @utp.ac.pa

\section{Introducción}

En el sistema educativo panameño aún prevalece el método de educación tradicional desde hace varias décadas. Este método se caracteriza por la presencia del profesor en el aula para explicar el tema de la clase y desarrollar ejemplos. Los estudiantes reciben la información, quizás tomando apuntes o tomando fotografías del tablero y prepararse para una prueba sumativa y unos meses después ya no recuerdan nada del tema. Para Roger C. Schank, uno de los factores por lo que sucede esto es porque no se aprende lo que nos dicen, se aprende lo que hacemos [1].
Esto lleva a la conclusión que el sistema educativo actual está planteado como una pirámide, de arriba hacia abajo, pero es preciso cambiar este método propio del siglo XIX y XX por un modelo asociativo: "alumnos y profesores tenemos que establecer alianzas, escucharnos unos a otros" [2].

Gracias a la internet, el conocimiento ya no es exclusivo de los profesionales universitarios que tuvieron acceso a las literaturas de sus profesores, sino que todos tenemos acceso a aprender lo que nos interesa. Muchas veces los estudiantes pierden el interés en la clase porque saben que pueden buscar 
lo que necesitan en internet y no están a expensas de lo que aprendieron con el profesor o no.

Estudios realizados sugieren que la utilización de técnicas o métodos de enseñanza como la gamificación[3], [4], el aprendizaje basado en juegos [5], el aprendizaje afectivo [6], el aula invertida o el aprendizaje móvil [7] llegan a incidir de manera positiva en la mayoría de los estudiantes a la hora de generarles mayor iniciativa por el desarrollo de un tema, manteniendo el interés en seguir recibiendo la información y que a la larga se ve reflejado en altos índices de aprobación en las asignaturas.

Por la situación actual de pandemia por la COVID-19, en la Educación Superior en Panamá, así como en muchos países, tuvo que hacer la transición de las clases presenciales a no presenciales [8], este cambio brinda la oportunidad perfecta para que profesores implementen algunas de las características positivas de los métodos mencionados para tratar de mantener la iniciativa en los estudiantes que muchas veces pueden verse afectados por falta de concentración en las actividades educativas o desinterés debido a que, al darse las clases en las casas y muchas veces en el propio dormitorio, el entorno no propicia la seriedad necesaria que brinda un aula de clases.

Todos estos factores inciden para que los métodos de enseñanza en la Educación Superior sean replanteados y actualizados a la realidad que vivimos, para tratar de potenciar las capacidades del estudiante, no solo del conocimiento técnico-científico, sino más integral como el desarrollo de nuevas habilidades blandas [9], desarrollando investigaciones autodidactas y trabajos colaborativos con compañeros de clases para lograr objetivos o competencias planteadas por el docente o el plan de estudio.

El desarrollo de este trabajo se presenta todo lo relacionado con la problemática a resolver en la sección dos (2), la metodología de solución, las herramientas empleadas por los estudiantes y los aprendizajes obtenidos que se plantea en la sección tres (3), las conclusiones y recomendaciones en la cuatro (4), por último, lo que se considera como trabajo futuro.

\section{Problema por resolver}

El hecho que las Instituciones de Educación Superior (IES) se hayan visto en la necesidad de aceptar a la modalidad no presencial para dar las clases virtuales por la COVID-19, las llevó a un nuevo reto ¿Cómo lograr que los estudiantes aprendan, desaprendan y reaprendan?

Esta dificultad era de esperar, porque no se puede seguir dando las clases a distancia como si fueran presenciales, no es lo mismo ni se aprende igual, porque el ambiente de aprendizaje cambia y tanto el docente como el estudiante tienen que aprender, desaprender y reaprender.

Si esto es así ¿Cómo despertar el interés en los alumnos con las condiciones actuales? A pesar de la situación actual, este cambio brinda la oportunidad perfecta para que profesores implementen elementos o ítems de métodos educativos que se ajusten mejor a esta nueva realidad.

Por otro lado, el amplio desarrollo y popularización de las redes informáticas, como internet, ha propiciado que gran cantidad de información esté a disposición prácticamente de cualquier persona en el mundo. Esto, sin duda, ha conllevado a que se plantee en más de una ocasión la modernización de los métodos de enseñanza, tanto en escuelas y colegios, como en las IES.

La premisa principal es que el docente debe convertirse, más que en la fuente de conocimiento, en el facilitador que oriente al estudiante a alcanzar a través de las investigaciones propias de los diferentes temas que conciernen al desarrollo de una asignatura en particular las competencias requeridas para la formación de un profesional competente.

Le corresponde al docente verificar que los estudiantes hayan comprendido los temas, abordando en el aula virtual las temáticas, para ofrecer la oportunidad a que los alumnos puedan expresar sus inquietudes, ofreciendo respuestas y alternativas para que amplíen su búsqueda de soluciones con otras fuentes externas.

Nos planteamos introducir una técnica colaborativa para que los estudiantes del Curso de Teoría de Control I en la Facultad de Ingeniería Eléctrica (FIE) de la Universidad Tecnológica de Panamá (UTP) consoliden los conocimientos adquiridos y propongan herramientas que contribuyan en el aprendizaje de otros estudiantes en cursos posteriores y adquieran más compromisos con su aprendizaje y lo compartan con aquellos que le es posible transferir información.

\section{Técnica colaborativa}

El caso de estudio se implementó en el Curso de Teoría de Control I, en un salón de cuarenta estudiantes de cuarto año en la carrera de Licenciatura en Ingeniería Electromecánica en la FIE de la UTP, Campus Víctor Levi Sasso.

Para mejorar el proceso de enseñanza-aprendizaje, se introdujo el componente participativo y colaborativo de los estudiantes, donde ya no eran simplemente los receptores de la información, sino que debían investigar en la web, y complementar ambos conocimientos para crear una manera didáctica de aprender la asignatura por medio de diferentes herramientas informáticas y facilitarla a otros estudiantes.

Con la técnica se persigue los siguientes objetivos:

- Formular desafíos que generen el interés del estudiante en ofrecer respuestas.

- Buscar repuestas a dichos desafíos a través del trabajo grupal o en equipo, permitiendo las posibilidades de comparación y retroalimentación de conocimientos entre ellos.

- Reforzar los conceptos de aprendizajes, para su examen semestral y para su formación holística a largo plazo.

- Plasmar material didáctico que contribuya al proceso de aprendizaje de otros estudiantes. 
Como método de aprendizaje en el aula, se dividió el salón de clases en ocho (8) equipos de cinco (5) personas cada uno. A cada dos equipos se le asignó un problema diferente para resolver, relacionado con casos reales o estudios de caso, donde era necesario utilizar el conocimiento aprendido a lo largo del semestre y los conocimientos adquiridos a través de la búsqueda en internet. Se incluyó un desafío adicional que consistía en desarrollar voluntariamente la manera didáctica de aprender a resolver el problema mediante alguna técnica o herramienta informática o digital que fomentara una actividad educativa interactiva sencilla.

Los problemas consistieron en la obtención de un controlador que garantizase que el sistema propuesto cumple con las especificaciones requeridas. El método a utilizar para la resolución fue libre, por lo que resultó igual de válido utilizar métodos estudiados en clase u otros con la consideración que se debían explicar los procedimientos.

Los estudiantes trabajaron las cuatro últimas semanas del curso, porque ya contaban con el conocimiento previo que requerían y para que, a su vez, pudieran prepararse para el examen semestral como un repaso.

El trabajo fue supervisado por el docente, quien daba seguimiento del proceso y verificaba los avances de los estudiantes periódicamente para garantizar la solución correcta, y el alcance de los objetivos planteados.

Es importante resaltar que el reto adicional de formular la manera didáctica de aprender a resolver un problema no era obligatorio para los estudiantes, pero el puntaje asignado al desarrollo de esta, tampoco tenía una ponderación significativa para la evaluación final del curso, pues se habían asignado tres que representaban el $15 \%$ de la nota semestral y esta tarea era una de tres asignaciones del curso. Todos los estudiantes asumieron el reto de ofrecer su aporte para los estudiantes que realizarán el curso posteriormente.

En la figura 1, se muestran las herramientas que los estudiantes escogieron para enfrentar el reto. La selección de la herramienta realizada por los estudiantes se hizo previa verificación que los estudiantes habían resuelto correctamente el problema asignado.

En las tablas 1, 2 y 3, se presentan las características de las herramientas utilizadas por los estudiantes para lograr hacerlas interactivas, es decir, Matlab [10], Power Point y Kahoot [11].

A los estudiantes se les suministró un conjunto de herramientas a su consideración y fue importante indicar que se les ofreció la opción de elegir la que ellos consideraban más apropiadas para desarrollar su trabajo, como efectivamente hicieron algunos equipos.

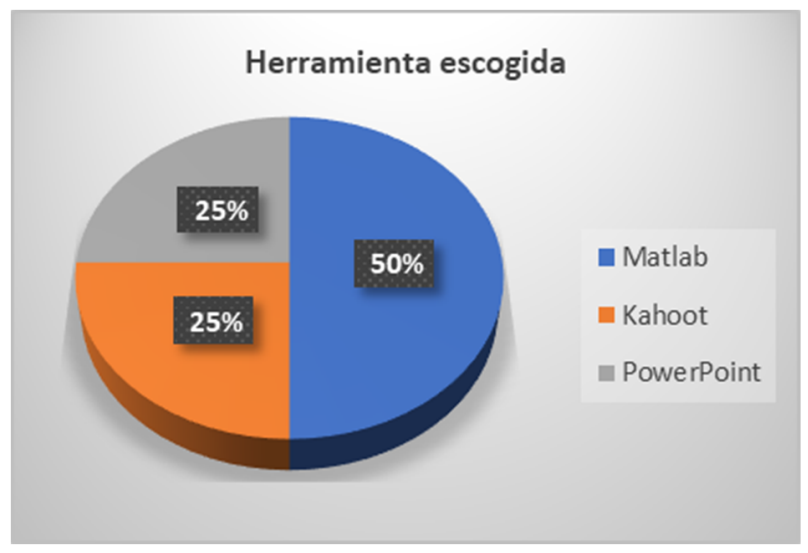

Figura 1. Herramientas para la interactividad

Se observa solo tres programas seleccionados para realizar el trabajo, porque se requería su dominio para aprender el manejo de nuevas herramientas que no era posible en el tiempo disponible de desarrollo.

Gracias a que el trabajo era en equipo, fue posible la realimentación entre compañeros; se verificaron los resultados; se discutió el método a utilizar para el diseño; se consensuó con el docente la mejor manera de presentar el problema a los demás estudiantes.

Tabla 1. Herramienta Matlab

\begin{tabular}{|c|c|}
\hline $\begin{array}{l}\text { Aplicación o } \\
\text { herramienta }\end{array}$ & Características principales \\
\hline Matlab & $\begin{array}{l}\text { - Lenguaje de alto nivel para } \\
\text { cálculos científicos y de } \\
\text { ingeniería. } \\
\text { Entorno de escritorio optimizado } \\
\text { para la exploración iterativa, el } \\
\text { diseño y la solución de } \\
\text { problemas. } \\
\text { Gráficas para visualizar datos y } \\
\text { herramientas para crear } \\
\text { diagramas personalizados. } \\
\text { Aplicaciones para ajustar curvas, } \\
\text { clasificar datos, analizar señales, } \\
\text { ajustar sistemas de control y } \\
\text { muchas otras tareas. } \\
\text { Toolboxes complementarias para } \\
\text { una amplia variedad de } \\
\text { aplicaciones científicas y de } \\
\text { ingeniería. } \\
\text { Herramientas para crear } \\
\text { aplicaciones con interfaces de } \\
\text { usuario personalizadas. } \\
\text { Interfaces para C/C++, } \\
\text { Java®, NET, Python, SQL, } \\
\text { Hadoop Microsoft Excel®. } \\
\text { Opciones de implementación } \\
\text { libres de derechos para compartir } \\
\text { programas de MATLAB con los } \\
\text { usuarios finales. }\end{array}$ \\
\hline
\end{tabular}


En general, cada equipo de trabajo se decidió por la creación de una especie de juego diferente, donde los participantes pudieran escoger la mejor respuesta y obtener una puntuación o estímulo. Se le diseñó un mecanismo de ayuda, y de retroalimentación para que el estudiante pudiera conocer la respuesta correcta en caso de cometer algún error.

A diferencia de otras ocasiones, donde era posible trabajar las asignaciones grupales reunidos de forma presencial para emitir de manera inmediata los aportes, por tratarse de clases a distancia, las aportaciones se hacían a manera de realimentaciones mutuas por medio de la utilización de plataformas de videollamadas y de aplicaciones de mensajería instantáneas.

Tabla 2. Herramienta Power Point

\begin{tabular}{|c|c|}
\hline $\begin{array}{l}\text { Aplicación o } \\
\text { herramienta }\end{array}$ & Características principales \\
\hline PowerPoint & 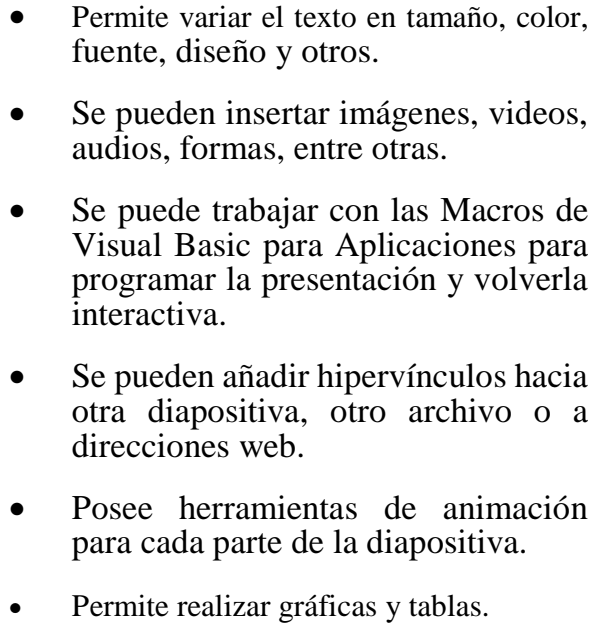 \\
\hline
\end{tabular}

Por los resultados obtenidos, como hubo pocos desaprobados, la percepción del profesor es que la implementación de este reto logró consolidar y fortalecer los conocimientos adquiridos a lo largo del semestre.

Esto es así, porque era necesario la adición del diseño de un controlador para asegurar el correcto funcionamiento del sistema dado. Para este estudio las condiciones requeridas eran imprescindible la base dada durante el semestre o simplemente repasarla.

Un aspecto importante que evidencia las estrategias para cumplir con la asignación por parte de los estudiantes es que, para compaginar la realización de este proyecto con el resto de las clases en el semestre, se le asignaron roles dentro del equipo, mientras unos eran los encargados de la resolución del problema con la herramienta seleccionada, para obtener las gráficas y demás resultados, otros se encargaban de la creación del juego tanto en la parte estética como funcional.
Todos trabajaron juntos, pero en actividades diferentes para lograr el objetivo común de presentar el producto final para la fecha indicada por el profesor. En otras palabras, acondicionaron su ambiente de aprendizaje [12].

Obsérvese que las tres tablas de las herramientas escogidas por los estudiantes fueron fácilmente utilizables por su sencillez, porque las dominaban, por la facilidad de agregarles animación y de diseñar.

Tabla 3. Herramienta Kahoot

\begin{tabular}{|c|c|}
\hline $\begin{array}{l}\text { Aplicación o } \\
\text { herramienta }\end{array}$ & Características principales \\
\hline Kahoot & $\begin{array}{l}\text { - Es flexible: Se ajusta a cualquier } \\
\text { temática de las diferentes asignaturas } \\
\text { del proceso de enseñanza aprendizaje, } \\
\text { que funciona como una herramienta de } \\
\text { evaluación. } \\
\text { Motiva el aprendizaje: Despierta el } \\
\text { interés del educando, porque se } \\
\text { generan procesos de interacción } \\
\text { activos, donde reflexionan para acertar } \\
\text { en la respuesta correcta. } \\
\text { Es de uso sencillo: Es una de las } \\
\text { plataformas educativas que no son } \\
\text { complejas al momento de utilizar. } \\
\text { Es global: Permite conectarnos a las } \\
\text { diferentes temáticas para poder jugar, } \\
\text { hasta encontrar distintos Kahoots en los } \\
\text { diferentes idiomas. } \\
\text { Es de Software gratuito: Es un servicio } \\
\text { web que ofrece acceso con la parte } \\
\text { gratuita que facilita para crear y hacer } \\
\text { uso de los cuestionarios,tiene su parte } \\
\text { Premium, con costo. }\end{array}$ \\
\hline
\end{tabular}

Quedan muchas interrogantes en el camino:

¿Podrá ser probada esta técnica con un nuevo grupo? ¿Se podrán generar estadísticas en el tiempo? ¿Será considerado este aporte estudiantil por la facultad para introducirlo en los cursos una vez probada la técnica? Estas preguntas y otras que salgan requieren de la continuidad de la investigación.

Aunque quedaron preguntas por responder, con el método utilizado se lograron diferentes objetivos, tales como:

- Reforzar los conocimientos previos, que implicó la búsqueda adicional de ejemplos y métodos por parte de los estudiantes.

- Desarrollar habilidades blandas como el trabajo en equipo, práctica de redacción de informes y creatividad. Esto fue así porque debieron sustentar sus propuestas. 
- Manejar conflictos, porque el equipo de trabajo se convirtió en una fuente de aprendizaje para el manejo de conflictos.

- Despertar el interés de los estudiantes en mejorar las técnicas y métodos de aprendizaje por ellos.

- Proponer herramientas de aprendizaje mediante la solución de problemas lo más cercano a la realidad.

\section{Conclusiones y recomendaciones}

El caso de estudio desarrolla acciones para el beneficio de los estudiantes, brindándoles facilidades para adquirir experiencias, profundizar los conceptos y reforzar los conocimientos.

El método utilizado, el aprendizaje colaborativo y con base a resolver problemas, estimula el interés de los estudiantes en participar, principalmente porque ponen en práctica lo aprendido.

Con el método utilizado se logra que el estudiante domine mejor la técnica de resolución de problemas, pueda demostrar detalladamente los procedimientos utilizados para resolver la problemática que se les asignó, y a la vez le facilita un modelo de trabajo a otros estudiantes que pueden aprender siguiendo estos procedimientos.

El método le facilita al estudiante organizar su tiempo para la solución del problema, coordinar con los demás miembros del grupo y ser autodidacta.

Formamos parte del estudio de caso, por este motivo recomiendo el uso de nuevas técnicas didácticas que estimulen al estudiante a poner en práctica sus conocimientos previos, a utilizar los recursos que nos ofrece la Web y proponernos un objetivo como: Propiciar material didáctico a los estudiantes que están en otros niveles.

Recomiendamos que en el período post COVID-19, la cultura académica permita que los ambientes de aprendizaje faciliten que las tecnologías educativas sean herramientas facilitadoras del aprendizaje, donde el docente sea guía del aprendizaje y un gestor de los tiempos que compartirá, ya sea en las modalidades presenciales, a distancia o semipresencial.

\section{Trabajo futuro}

Uno de los aspectos importantes en este tipo de trabajo es la verificación de las estrategias de aprendizaje diseñadas por estudiantes para otros grupos de clases que tengan los resultados deseados para darle continuidad.

Por ello, planteamos para el futuro:

- Realizar algún tipo de comparación con otro grupo que no sea presencial y que no emplee este método, porque fue la primera vez que se impartió el curso en esta modalidad.

- Que el profesor implemente el método en el siguiente año académico para que se generen otros grupos del curso.
- Que otro grupo no presencial realice algún tipo de evaluación de los problemas diseñados por los nuevos estudiantes.

- Verificar con otro grupo no presencial si los trabajos hechos por los estudiantes del curso de Teoría de Control anterior son lo suficientemente motivadores para ellos.

- Sería interesante introducir nuevas técnicas y sean evaluadas por los estudiantes.

\section{REFERENCIAS}

[1] M. López Blanco, "Los colegios no deberían existir," Kindsein Magazine, 2007. [Online]. Available: http://www.kindsein.com/es/21/1/485/. [Accessed: 01-Nov2020].

[2] R. Calleja, "Un nuevo modelo educativo para el siglo XXI," Comunidad Esc. periódico Digit. Inf. Educ., vol. 880, pp. 65-134, 2010.

[3] M. C. Sierra Daza and M. R. Fernández-Sánchez, "Gamificando el aula universitaria. Análisis de una experiencia de Escape Room en educación superior,” Rev. Estud. y Exp. en Educ., vol. 18, no. 36, pp. 105-115, Apr. 2019.

[4] I. A. Hernández-Horta, A. Monroy-Reza, and M. Jiménez-García, "Aprendizaje mediante Juegos basados en Principios de Gamificación en Instituciones de Educación Superior," Form. Univ., vol. 11, no. 5, p. 31, 2018.

[5] J. L. Gómez Contreras, "Gamificación en contextos educativos: análisis de aplicación en un programa de contaduría pública a distancia," Rev. Univ. y Empres., vol. 22, no. 38, 2020.

[6] D. S. Melo-Solarte and P. A. Díaz, "El Aprendizaje Afectivo y la Gamificación en Escenarios de Educación Virtual," Inf. Tecnol., vol. 29, no. 3, Jun. 2018.

[7] E. Fernández and A. Arcos, "Objetos de aprendizaje, aula invertida, gamificación y m-learning para la enseñanza de medicina en la Universidad UNIANDES," Pontificia Universidad Católica del Ecuador. Sede Ambato. Repositorio PUCESA. 2017.

[8] N. Archer Svenson and G. De Gracia, "Educación Superior Y Covid-19 En La República De Panamá,” ESAL - Rev. Educ. Super. en América Lat., pp. 15-19, 2020.

[9] M. Espinoza M. and D. del P. Gallegos B., "Habilidades blandas y su importancia de aplicación en el entorno laboral: perspectiva de alumnos . de una universidad privada en Ecuador," 2020.

[10] MATLAB \& Simulink, "Descripción del producto MATLAB MATLAB \& Simulink - MathWorks América Latina.” 2020.

[11] P. S. Yugcha Lincango, "Kahoot en el proceso de enseñanza aprendizaje en la asignatura de Biología en los Primeros de Bachillerato General Unificado de la Unidad Educativa Municipal del Milenio 'Bicentenario', período 2019-2020," Universidad Central del Ecuador, Quito, 2020.

[12] Pastor, A. y Chiappe, A. "Papel de los Mundos Metaversos como alternativa de generación de condiciones clave en la construcción de Ambientes Personales de Aprendizaje," Universidad de la Sabana. 2014. Colombia. 